\title{
Application of the minimum state error variance approach to nonlinear system control
}

\author{
L. M. Miškovićł§, Ž. M. Duurovićł and B. D. Kovačevićłł*
}

\begin{abstract}
A class of modified state space self-tuning controllers of the minimum state error variance type was considered. A suitable chosen structure of the proposed controller allows the tracking of a time-varying reference input and makes a possibility of applying this solution to nonlinear and non-stationar y plants. The advantage in using the proposed algorithm for nonlinear systems' control is demonstrated through its application to aircraft control around a prespecified reference trajectory in the presence of characteristic disturbances. The results show that the proposed controller has good tracking performance and possesses rather good immunity towards disturbances.
\end{abstract}

\section{Introduction}

Two groups of optimization-based adaptive controllers have drawn wide attention in recent years and have been widely studied in the literature. The first makes use of the state space representation of the system coupled with the linear quadratic Gaussian (LQG) optimal control theory and a sequential parameter estimation technique to obtain an adaptive filtered state feedback controller (Anderson and Moore 1979, Åström 1970, Åström and Wittenmark 1989, 1990). The optimal adaptive control algorithms obtained have the advantage of being globally stable, of being applicable to any finite dimensional controllable and observable system, and of providing with an effective control of the errors in the state trajectories. These are, however, achieved at the cost of a rather large computational burden, which makes it difficult to implement these algorithms in real-time for some

Received 27 March 2000. Revised 6 November 2001. Accepted 20 December 2001

$\dagger$ Control Systems Department, Faculty of Electrical Engineering, University of Belgrade, Bulevar Kralja Aleksandra 73, 1100 Belgrade, Yugoslavia.

$\ddagger$ Instituto Superior Tecnico, Instituto de Sistemas e Robotica, Torre Norte, 6 Piso IST-Av. Rovisco Pais,1049-00 1 Lisbon, Portugal

$\S$ Currently with the Automatic Institute of Swiss Federal Institute of Technology at Kaussane (EPEL), Switzerland.

- Currently with Visteon Automotive Systems, Advanced Technology Europe.

* To whom correspondence should be addressed. e-mail: kovacevic_b@kiklop.etf.bg.ac.yu practical systems. The second group makes use of the input-output representation of system, coupled with the minimization of a generalized output error variance (Clarke and Gawthrop 1975, Grimble 1981, Warwick 1981). The main advantage of such a type of controllers, named self-tuning controllers, includes the relative simplicity of their derivation and implementation. However, the performance index selected for this approach does not minimize the errors in the state trajectories, as may be required in some applications. Also, the global stability of the controlled system requires the inverse system to be stable, which may exclude some non-minimum phase systems (Grimble 1981). A class of state space self-tuning controllers, named the minimum state error variance (MSEV) algorithm, that represents a combination of the mentioned approaches has been presented (Desai and Mahanalabis 1991, Mišković 1999, Mišković et al. 2000). The MSEV controller is analogous in the form to the LQG optimal adaptive controller, but it achieves a considerable reduction of the computational requirement at the cost of some performance loss.

Extensions of the MSEV approach to the control of nonlinear and non-stationary plants are proposed here. In contrast to the original MSEV approach, these extensions provide for tracking a prespecified nominal state space trajectory in the presence of stochastic disturbances with unknown statistics. The feasibility of the proposed control strategy has been demonstrated through its application to a six degrees-of-freedom aircraft model. 


\section{Problem formulation}

Consider the system:

$$
\begin{aligned}
x(k+1) & =\Phi x(k)+\Psi u(k)+\Gamma w(k) \\
y(k) & =H x(k)+v(k),
\end{aligned}
$$

where $x(k) \in R^{n}$ is the state, $u(k) \in R^{m}$ is the input and $y(k) \in R^{p}$ is the output of the system, $\{w(k)\}$ and $\{v(k)\}$ are the zero-mean disturbance terms with covariance matrices $Q$ and $R$, respectively, and $\Phi, \Psi, \Gamma$ and $H$ are the known system matrices. Then introduce the single stage performance index:

$$
\begin{aligned}
J(u) & =E\{L[x(k+1), u(k)]\}, \\
L[x(k+1), u(k)] & =\left[\begin{array}{c}
x(k+1) \\
u(k)
\end{array}\right]^{\mathrm{T}}\left[\begin{array}{cc}
W & S \\
S^{\mathrm{T}} & U
\end{array}\right]\left[\begin{array}{c}
x(k+1) \\
u(k)
\end{array}\right],
\end{aligned}
$$

where $W$ and $S$ are constant, bounded and nonnegative definite weighting matrices $(W \geq 0, S \geq 0)$, while $U$ is a constant, bounded and positive definite weighting matrix $(U>0)$ (Desai and Mahanalabis 1991). The control obtained by minimizing (2) will be referred to as the MSEV control and is given by:

$$
\begin{aligned}
u(k) & =M \hat{x}(k) ; \hat{x}(k)=E\{x(k) \mid Y(k-1)\} \\
M & =-\left(\Psi^{\mathrm{T}} W \Psi+U+2 \Psi^{\mathrm{T}} S\right)^{-1}\left(\Psi W \Phi+S^{\mathrm{T}} \Phi\right),
\end{aligned}
$$

where $Y(k-1)=\{y(0), y(1), \ldots, y(k-1)\}$ is the set of measurements up to time $(k-1) T$, when $T$ is the sampling period. Here, $\hat{x}(k)$ represents the minimum variance one-step-ahead prediction of the state $x(k)$ and may be generated by using the well-known Kalman predictor, which requires the noise statistics $Q$ and $R$ to be known exactly (Anderson and Moore 1971, 1979, Åström 1970, Kovačević and Djurović 1999). However, if the system parameters of model (1) are unknown, the parameters have to be estimated recursively before the control algorithm (3) can be implemented. To ensure identifiability, model (1) is replaced by the steady-state innovations model in observer canonical form (Anderson and Moore, 1979, Ljung and Söderström 1983):

$$
\begin{aligned}
x(k+1) & =\Phi x(k)+\Psi u(k)+\Gamma e(k) \\
y(k) & =H x(k)+e(k),
\end{aligned}
$$

where the matrices $\Phi$ and $H$ are assumed to have the following form:

$$
\Phi=\left[\begin{array}{c|ccc}
-a_{1} & & & \\
\vdots & I_{(n-1) \times(n-1)} \\
-a_{n-1} & & & \\
-a_{n} & 0 & \cdots & 0
\end{array}\right] ;
$$

$$
H^{\mathrm{T}}=\left[\begin{array}{c}
1 \\
0 \\
\vdots \\
0
\end{array}\right] ; \Psi=\left[\begin{array}{c}
\psi_{1} \\
\vdots \\
\psi_{n}
\end{array}\right] ; \Gamma=\left[\begin{array}{c}
\gamma_{1} \\
\vdots \\
\gamma_{n}
\end{array}\right]
$$

with $I_{(n-1) \times(n-1)}$ being the identity matrix of the order $n-1$.

The problem of parameter estimation of the observer state space form (5) may be solved by taking advantage of the fact that the model may be equivalently expressed in the form of $n$-dimensional autoregressive moving average model with exogenous input, the so-called ARMAX model, given below (Anderson and Moore 1979, Goodwin and Sin 1984, Ljung and Söderström 1983):

$$
A\left(q^{-1}\right) y(k)=B\left(q^{-1}\right) u(k)+D\left(q^{-1}\right) e(k),
$$

where $q^{-1}$ is the unit delay operator. The polynomials $A$, $B$ and $D$ have the following forms:

$$
\begin{aligned}
& A\left(q^{-1}\right)=1+\sum_{i=1}^{n} a_{i} q^{-i} \\
& B\left(q^{-1}\right)=\sum_{i=1}^{n} b_{i} q^{-i} \\
& D\left(q^{-1}\right)=1+\sum_{i=1}^{n} d_{i} q^{-i} .
\end{aligned}
$$

The coefficients $a_{i}, b_{i}$ and $d_{i}, i=1, \ldots, n$ are easily obtained from the elements of the matrices $\Psi$ and $\Gamma$; that is (Ljung and Söderström 1983):

$$
\psi_{i}=b_{i} ; \quad \gamma_{i}=d_{i}-a_{i} ; \quad i=1, \ldots, n .
$$

Moreover, it is possible to express the ARMAX form (6) in the following linear regression form:

$$
y(k)=Z^{\mathrm{T}}(k) \Theta+e(k),
$$

where $\theta$ is the vector of unknown parameters given as:

$$
\Theta=\left[a_{1}, \ldots, a_{n}, b_{1}, \ldots, b_{n}, \gamma_{1}, \ldots, \gamma_{n}\right]^{\mathrm{T}}
$$

and $Z(k)$ is the regression vector containing appropriate set of past outputs, inputs and innovations:

$$
\begin{aligned}
Z(k)= & {[-y(k-1), \ldots,-y(k-n),} \\
& u(k-1), \ldots, u(k-n), \\
& e(k-1), \ldots, e(k-n)]^{\mathrm{T}} .
\end{aligned}
$$

Once the regression model (9-11) is obtained, a recursive estimation of the parameter vector $\Theta$ may be achieved using a number of alternative algorithms (Ljung and Söderström 1983, Goodwin and Sin 1984, Tsypkin 1984). Here, it is used the pseudolinear regression algorithm (Ljung and Söderström 1983): 


$$
\begin{aligned}
\hat{\Theta}(k)= & \hat{\Theta}(k-1)+G(k) \varepsilon(k) \\
\varepsilon(k)= & y(k)-Z^{\mathrm{T}}(k) \hat{\Theta}(k-1) \\
G(k)= & T(k-1) Z(k)\left[1+Z^{\mathrm{T}}(k) T(k-1) Z(k)\right]^{-1} \\
T(k)= & T(k-1)-T(k-1) Z(k) Z^{\mathrm{T}}(k) T(k-1) \\
& \times\left[1+Z^{\mathrm{T}}(k)(k-1) Z(k)\right]^{-1} .
\end{aligned}
$$

These equations are initialized with an assumed initial estimate $\hat{\Theta}(0)$ and initial covariance $T(-1)$ and may be expected to converge to the correct parameters under certain conditions (Ljung and Söderström 1983, Goodwin and Sin 1984). In this way, the adaptive MSEV controller is obtained easily from (3), where $\hat{x}(k)$ is replaced by $x(k)$ from (4), while the system matrices $\Psi, \Phi$ and $\Gamma$ are replaced by their estimates $\hat{\Psi}$, $\hat{\Phi}$ and $\hat{\Gamma}$ from (12), respectively. Furthermore, an asymptotic state prediction is achieved after direct estimation of the parameters of the innovations model (5), without requiring an explicit knowledge of the noise covariances $R$ and $Q$. The major computational requirements of the MSEV controller arise from the parameter estimation algorithm (12), which is central to the implementation of any form of adaptive controller (Åström and Wittenmark 1989, 1990, Goodwin and Sin 1964).

\section{Adaptive MSEV controller for nonlinear systems}

The MSEV method discussed above may be extended so to design an estimated state feedback controller for nonlinear and non-stationary systems with non-zero reference signal. The scheme for such implementation of the MSEV controller, named modified MSEV controller, is shown in figure 1 . Here, $u_{\text {ref }}(k)$ and $y_{\text {ref }}(k)$ represent a given time-varying deterministic reference, or nominal trajectory, while $u(k)$ and $y(k)$ are deviations from the nominal signals $u_{\text {ref }}(k)$ and $y_{\text {ref }}(k)$, respectively.

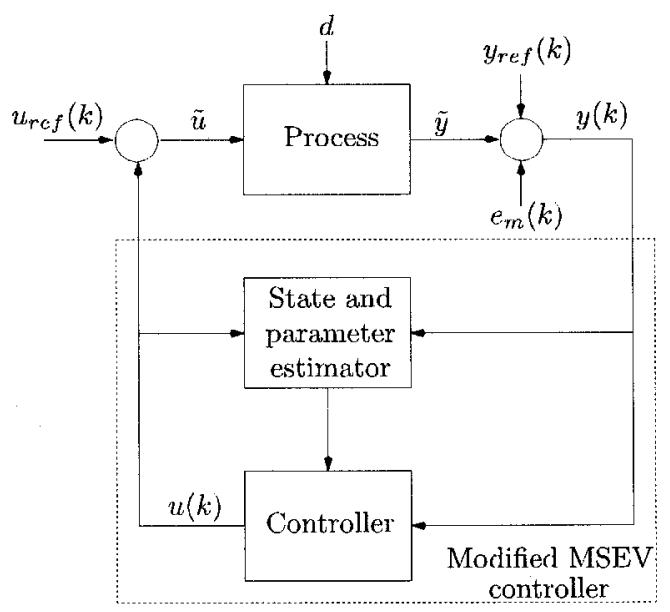

Figure 1. Modified adaptive MSEV controller.
Moreover, $d$ and $e_{m}(k)$ denote the process and observation disturbance samples, respectively. In this way, the signal $u(k)$ generated by the controller represents a correction around the nominal, or reference, control signal. Thus, the adopted one structure of the control system enables one to track the reference trajectory of a desired form. Furthermore, a since the MSEV approach uses an online identification of linear system (a linear system describes a plant in the vicinity of a point on the nominal trajectory), it can also be applied to nonlinear systems, after linearizing the nonlinear system characteristics around the properly chosen operating points. The choice of operating points should be done in a such way to cover characteristic nonlinear regimes of the system concerned. In this way, various linear models will describe the behaviour of the nonlinear system in the vicinity of the chosen operating points. Finally, starting from the fact that linearized model parameter estimation is performed online, this approach is also appropriate for control of non-stationary systems, provided that the dynamics of these non-stationarities are negligible compared with the system dynamics.

In practice, the reference trajectory is usually obtained either by developing a complex nonlinear model of the system in question or by simulation under some reasonable operating conditions. Adopted here is numerical approach based on the strategy of predictive control and optimization under constraints (i.e. $u_{\min } \leq u_{\text {ref }} \leq u_{\max }$, where bounds $u_{\min }$ and $u_{\max }$ have to be determined on the basis of known process properties).

Let $y_{\text {ref }}^{\text {nom }}$ represent the desired nominal trajectory, then the nominal control signal $u_{\text {ref }}$, at stage $k$, can be calculated to minimize the deflection from the desired nominal trajectory, i.e.

$$
\begin{aligned}
u_{\mathrm{ref}}(k T)= & \arg \left\{\operatorname { m i n } _ { u _ { \mathrm { ref } } } \left(y_{\mathrm{ref}}\{(k+N) T\}\right.\right. \\
& \left.\left.-y_{\mathrm{ref}}^{\text {nom }}\{(k+N) T\}\right)^{2}\right\},
\end{aligned}
$$

where $y_{\text {ref }}\{(k+N) T\}$ denotes the $y_{\text {ref }}$-coordinate at the instant $(k+N) T$. The sequence $y_{\text {ref }}\{(k+N) T\}$ is obtained by solving the corresponding nonlinear statespace model of the system concerned under the condition that the control signal $u_{\text {ref }}(t)$ is constant over $N$ consecutive sampling periods, $k, k+1, \ldots, k+N-1$, respectively. Figure 2 represents the flowchart of the proposed algorithm for calculating the desired reference control signal under which the desired reference output trajectory will be achieved. One can use, for example, the Runge-Kutta method of the fourth or fifth order for obtaining the state trajectory and the response of a nonlinear system model in question. Furthermore, a gradient-type procedure for solving the optimization problem (13) can also be used. Particularly, the 


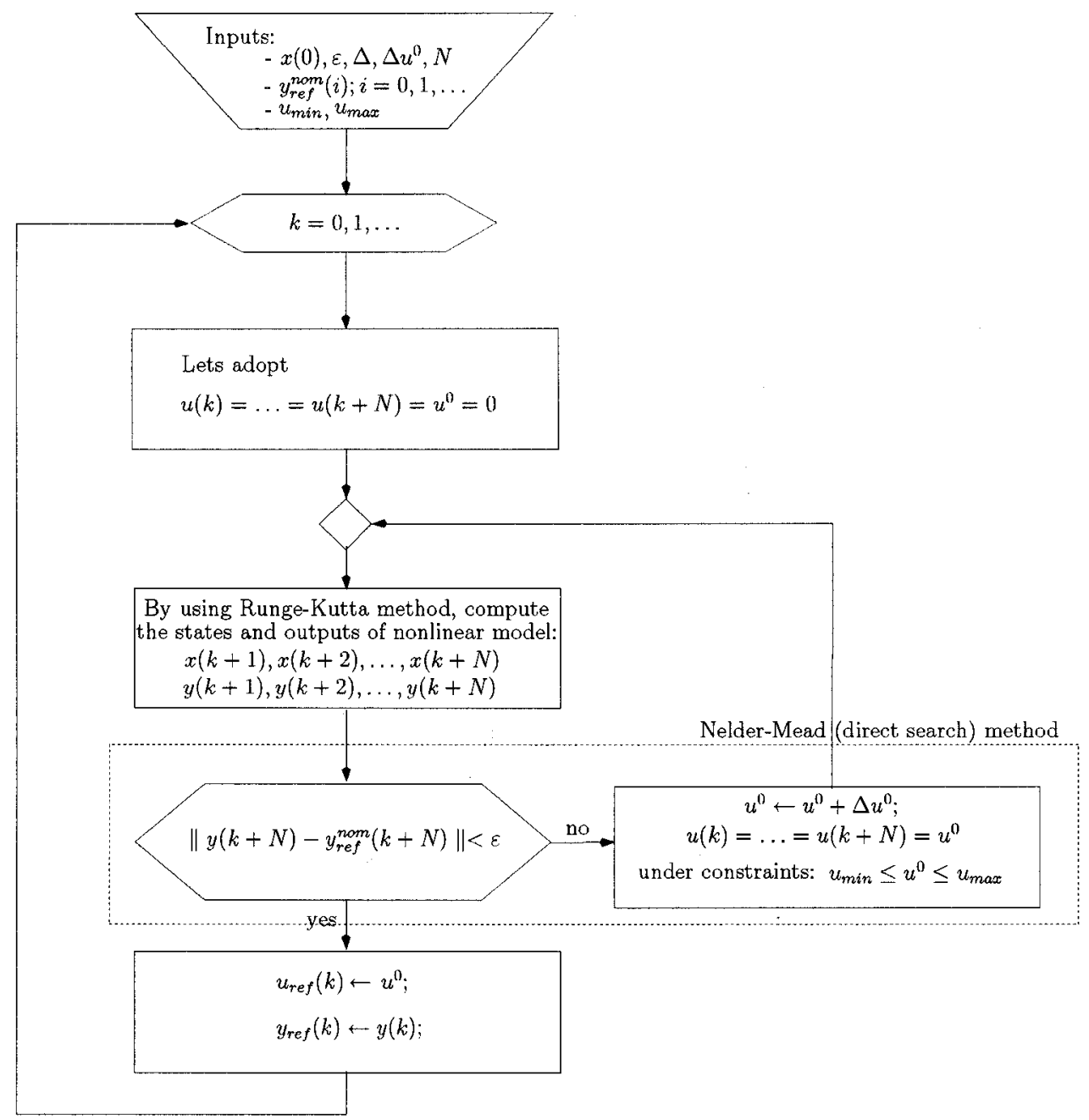

Figure 2. Calculation of the nominal control signal.

Nelder-Mead direct search method represents a good procedure to be used for this purpose (Dixon 1972).

Prediction horizon $N$ represents a free parameter, which has to be adopted in advance. The choice of $N$ represents a compromise between two opposite requirements concerning the allowable values and dynamics of the reference control input $u_{\text {ref }}$, and the corresponding admissible errors in tracking the prespecified reference trajectory. A smaller $N$ will result into the control signal $u_{\text {ref }}$ very close to the prespecified bounds to minimize as fast as possible the deflections from the nominal trajectory. On the other hand, higher $N$ will result in the control signal with smaller dynamics, which is not influenced too much by the given control bounds. However, this will yield rather large deflections from the given nominal trajectory $y_{\text {ref }}^{\text {nom }}$ as it will be shown below.

To demonstrate the feasibility of the approach, consider the example of an aircraft control around the state reference trajectory, as given in Koifman and Merhav (1991).

\section{Simulated example}

An aircraft motion is described by 12 standard nonlinear differential equations given below, known in the literature as the six degrees-of-freedom (6DOF) flight model (Koifman and Merhav 1991):

(1) Dynamical translatory equations in body axes:

$$
\begin{aligned}
\dot{u}= & -\frac{\rho V^{2}}{2}\left[C_{D} \cos (\alpha) \cos (\beta)+C_{Y} \cos (\alpha) \sin (\beta)\right. \\
& \left.+C_{L} \sin (\alpha)\right]+\frac{T_{\mathrm{m}}}{m}-g \sin (\theta)-g w+r v \\
\dot{v}= & -\frac{\rho V^{2}}{2}\left[-C_{D} \sin (\beta)+C_{Y} \cos (\beta)\right] \\
& +g \sin (\phi) \cos (\theta)-r u+p v \\
\dot{w}= & \frac{\rho V^{2}}{2}\left[C_{D} \sin (\alpha) \cos (\beta)+C_{Y} \sin (\alpha) \sin (\beta)\right. \\
& \left.-C_{L} \cos (\alpha)\right]-g \cos (\theta) \sin (\phi)-p v+g u .
\end{aligned}
$$


(2) Dynamical angular equations in body axis:

$$
\begin{aligned}
\dot{p} & =C_{t} \frac{1}{2} \rho V^{2} S d / A \\
\dot{q} & =\left(C_{m} \frac{1}{2} \rho V^{2} S d / B+(C-A) r p\right) / B \\
\dot{r} & =C_{n} \frac{1}{2} \rho V^{2} S d / C+((A-B) p q) / C .
\end{aligned}
$$

(3) Kinematical (Euler) equations:

$$
\begin{aligned}
& \dot{\phi}=p+(r \cos (\phi)+q \sin (\phi)) \tan (\theta) \\
& \dot{\theta}=q \cos (\phi)-r \sin (\phi) \\
& \dot{\psi}=(r \cos (\phi)+q \sin (\phi)) / \cos (\theta) .
\end{aligned}
$$

(4) Transformation of ground speed from body to Earth coordinates:

$$
\begin{aligned}
\dot{X}= & u \cos (\theta) \cos (\psi)+v(\sin (\psi) \sin (\theta) \cos (\psi) \\
& -\cos (\phi) \sin (\psi))+w(\cos (\phi) \sin (\theta) \cos (\psi) \\
& +\sin (\phi) \sin (\psi)) \\
\dot{Y}= & u \cos (\theta) \sin (\psi)+v(\sin (\phi) \sin (\theta) \sin (\psi) \\
& +\cos (\phi) \cos (\psi))+w(\cos (\phi) \sin (\theta) \sin (\psi) \\
& -\sin (\psi) \cos (\psi)) \\
\dot{Z}= & -u \sin (\theta)+u \sin (\phi) \cos (\theta)+w \cos (\phi) \cos (\theta) .
\end{aligned}
$$

Here, $\rho$ is air density, $S$ is the reference of the crosssectional area, $d$ is body diameter, $m$ is aircraft mass, $g$ is Earth acceleration and $T_{\mathrm{m}}$ is thrust moment.

The absolute aircraft speed $V$, angle of attack $\alpha$ and angle of sideslip $\beta$ are given by:

$$
\begin{aligned}
& V=\sqrt{u^{2}+v^{2}+w^{2}} \\
& \alpha=\tan ^{-1}(w / u) ; \quad \beta=\tan ^{-1}(v / u) .
\end{aligned}
$$

The parameters $A, B$ and $C$ in equations (17-19) are time varying, since they are dependent on the aircraft mass and drag force, while the aerodynamic coefficients $C_{D}, C_{Y}, C_{L}, C_{m}, C_{n}$ and $C_{l}$ represent the functions of angles $\alpha$ and $\beta$, the angular velocities $p, q, r$ and the angles $\delta_{\mathrm{A}}, \delta_{\mathrm{E}}$ and $\delta_{\mathrm{R}}$. The functional relations between these quantities, as well as the real values of the parameters characterizing the 6DOF model, are given in Koifman and Merhav (1991). The last three angles are the input variables satisfying the boundary condition $|\delta| \leq 0.25 \mathrm{rad}$. Thus, the system of nonlinear differential equations (14-27) can be represented in the nonlinear state space form

$$
\dot{x}=f(x(t), u(t)) .
$$

Here $x(t) \in R^{12}$ is the state vector defined by

$$
x(t)=[u, v, w, p, q, r, \phi, \psi, \theta, X, Y, Z]^{\mathrm{T}}
$$

and $u(t) \in R^{3}$ is the control vector given by

$$
u(t)=\left[\delta_{\mathrm{A}}, \delta_{\mathrm{E}}, \delta_{\mathrm{R}}\right]^{\mathrm{T}},
$$

where the three elements stand for aileron, elevator and rudder, respectively. In addition, $f($.$) is the corre-$ sponding 12th dimensional nonlinear vector function, describing the nonlinear system dynamics. The reference trajectory, presented in figure 3 , is specified in the $(X, Z)$ plane taking into account the flight conditions and the real capabilities of the aircraft.

Additionally, it is necessary to calculate the nominal control $u_{\text {nom }}=\left[\delta_{\mathrm{A}}^{\text {nom }}, \delta_{\mathrm{E}}^{\text {nom }}, \delta_{\mathrm{R}}^{\text {nom }}\right]$ across the given reference trajectory $Z^{\text {nom }}$. However, since the trajectory is defined in the vertical plane $(Y=0)$, it is natural to choose $\delta_{\mathrm{R}}^{\text {nom }}=0$ and $\delta_{\mathrm{A}}^{\text {nom }}=0$. Thus, the 12th-order nonlinear system (28) with the one input $u_{\text {nom }}(t)=\delta_{\mathrm{E}}^{\text {nom }}(t)$ and the one output $y_{\text {nom }}(t)=Z^{\text {nom }}(t)$ is defined. The nominal control $\delta_{\mathrm{E}}^{\text {nom }}$ is calculated using the strategy of predictive control (figure 2), described by (13). Namely, it is assumed that the control signal $\delta_{\mathrm{E}}(t)$ is constant within the several sampling periods (the sampling period is chosen to be $T=0.5 \mathrm{~s}$ ), and then the nominal control is calculated to minimize the deflection from the specified nominal state trajectory, i.e.

$$
\delta_{\mathrm{E}}^{\text {nom }}(k T)=\arg \left\{\min _{\delta_{E}}\left[Z((k+N) T)-Z^{\text {nom }}((k+N) T)\right]^{2}\right\} .
$$

As mentioned above $Z((k+N) T)$ denotes the value of $Z$-coordinate at the instant $(k+N) T$ obtained by solving the nonlinear state-space equation (28) under the condition that the control signal $\delta_{\mathrm{E}}(t)$ is constant over the $N$ consecutive sampling periods $k, k+1, \ldots, k+N-1$, respectively, and $\delta_{\mathrm{R}}=\delta_{\mathrm{A}}=0$. The prediction horizon $N$ represents a free parameter that must be chosen in advance. Figures 4 and 5 depict the control signal $\delta_{\mathrm{E}}$ for different values of the prediction horizon $N$, while figures 6 and 7 show the

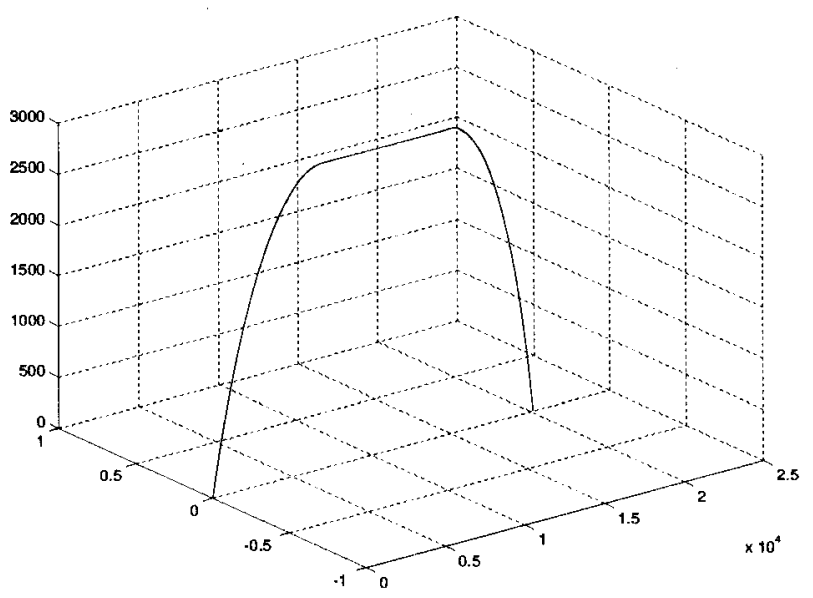

Figure 3. Nominal trajectory. 


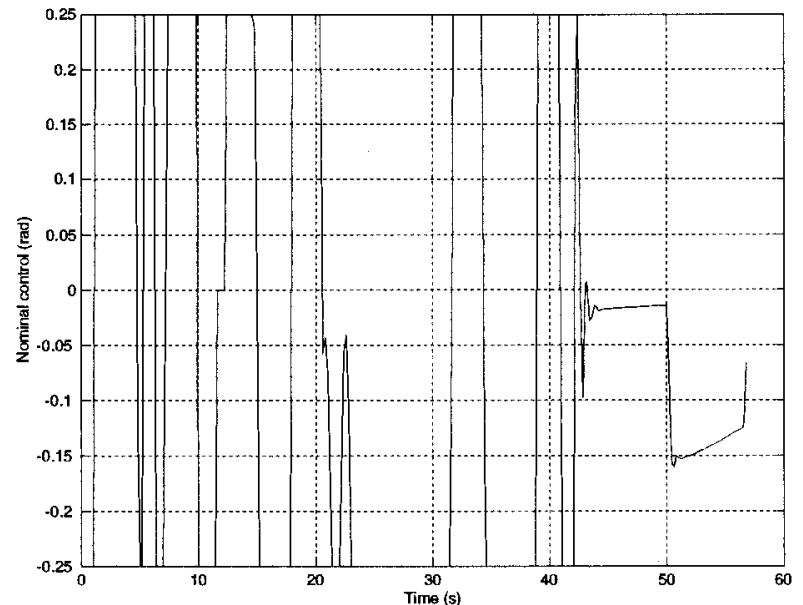

Figure 4. Control signal for prediction horizon, $N=2$.

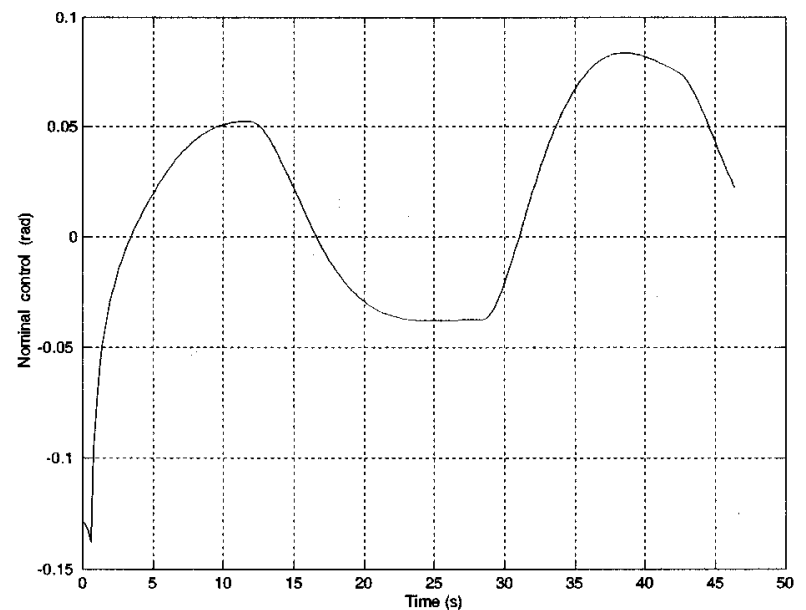

Figure 5. Control signal for the prediction horizon, $N=\mathbf{2 0}$.

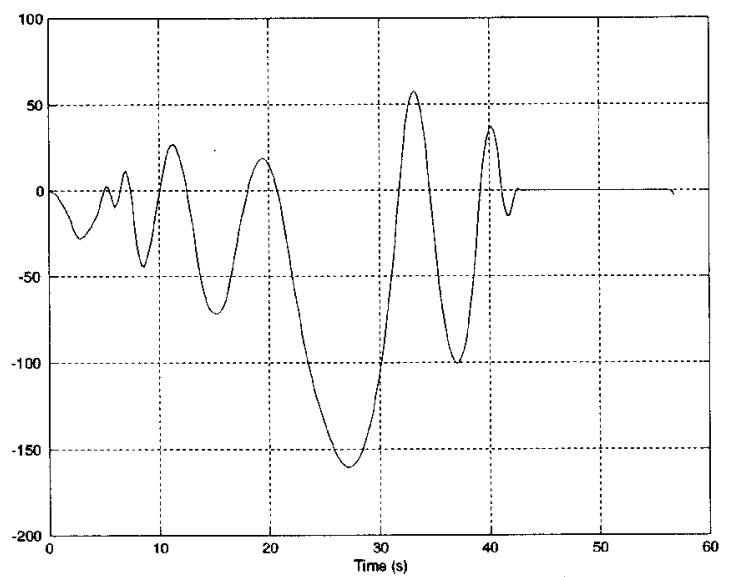

Figure 6. Deflection from the nominal trajectopry for the prediction horizon, $N=2$.

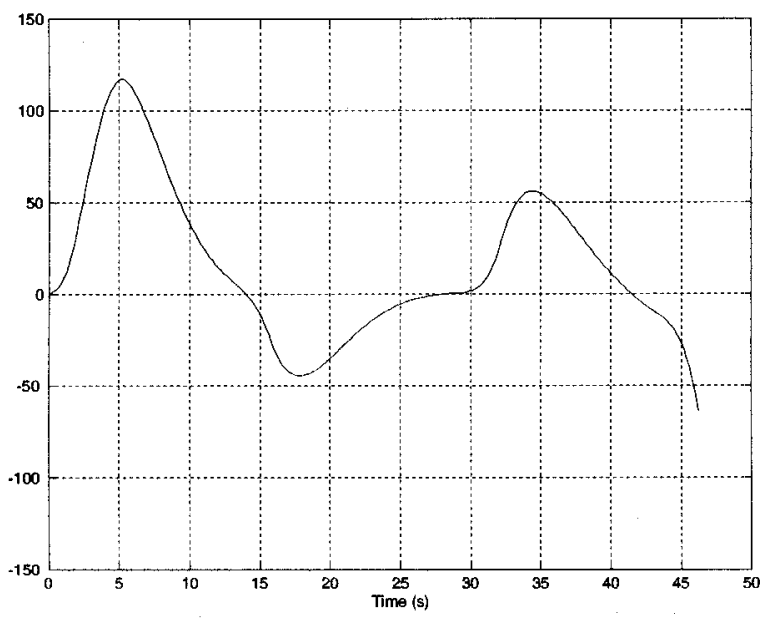

Figure 7. Deflection from the nominal trajectory for the prediction horizon, $N=20$.

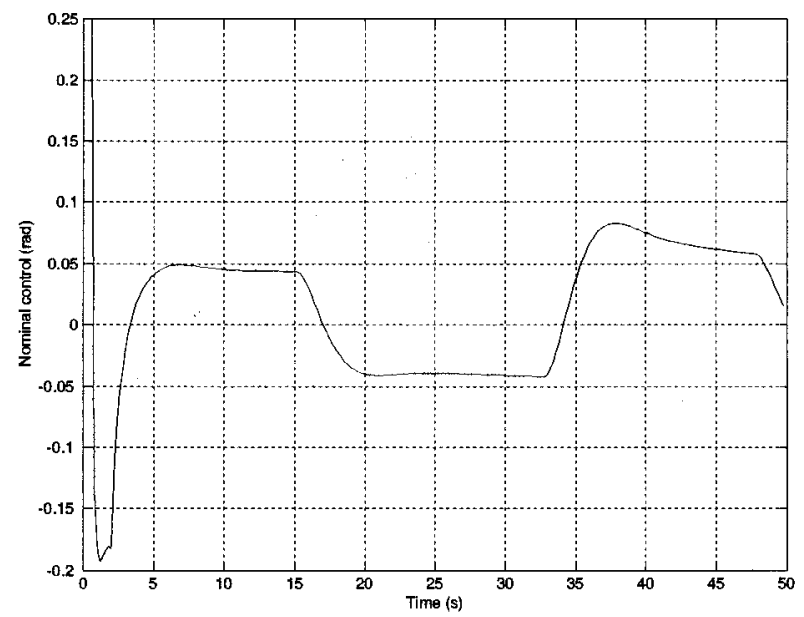

Figure 8. Nominal control for the prediction horizon, $N=4$.

corresponding deflections from the nominal trajectory. As a result of this brief experimental analysis, it is chosen as $N=4$. The calculated nominal control for the chosen horizon of prediction is given in figure 8 .

To perform the control task, in the sense that the system output $y(k)=Z(k)$ is to be forced by the input $u(k)$ to track the reference output $y^{\text {nom }}(k)=Z^{\text {nom }}(k)$ in the presence of additive measurement noise $e_{\mathrm{m}}$, the MSEV controller is used (figure 1).

The measurement noise $e_{\mathrm{m}}$ is supposed to be a zeromean stationary white Gaussian sequence with variance $\sigma_{0}^{2}=50$. The output signal $y(k)=Z(k)$, corresponding to the nominal input $\delta_{\mathrm{E}}^{\text {nom }}$, is corrupted by the samples of specified measurement noise. As mentioned above, the design of the MSEV controller is based on the ARMAX model (9) of the order $n$. It is convenient to choose a lower order of a linearized system model for controller 
design purposes, which has to represent a good compromise between controller efficiency and its computational complexity. After a numerous simulations, it is adopted that the order of the ARMAX model is $n=4$. Furthermore, the weighting matrices $W, S$ and $U$ also represent the free parameters in the MSEV design. These matrices have to be chosen in advance, taking into account the ratio of the absolute values of the control signal $u=\delta_{\mathrm{E}}$ and the elements of the state vector in (29), as well as the given physical bounds on the control input and the requirements for the global asymptotical stability of the overall control system. In practice, these matrices can be chosen by simulation. As a result of such analysis, they are chosen

$$
\begin{aligned}
W & =\operatorname{diag}\{0.01,0,0,0\} \\
S & =0.02[1,1,1,1]^{\mathrm{T}} \\
U & =1000,
\end{aligned}
$$

where $\operatorname{diag}\{$.$\} is the diagonal matrix.$

To investigate the performance of the proposed adaptive controller, a comparison with the conventional fixed parameter controller is made. Figure 9 shows the errors in tracking the nominal trajectory for the adaptive controller, while figure 10 gives the same performance measure for the fixed parameter controller. Figures 11 and 12 show the corrections of the control signal $u(k)$ for these two controllers. Obviously, the deflections from the reference trajectory are significantly smaller in the case of a model parameter adaptation.

The central issue to the tracking capability of any adaptive control scheme is good convergence to the unknown model parameters. Figure 13 depicts the estimated $a$-parameters of the adopted fourth-order ARMAX model in (9-11) used for the adaptive controller design. Since the 12 th order nonlinear model

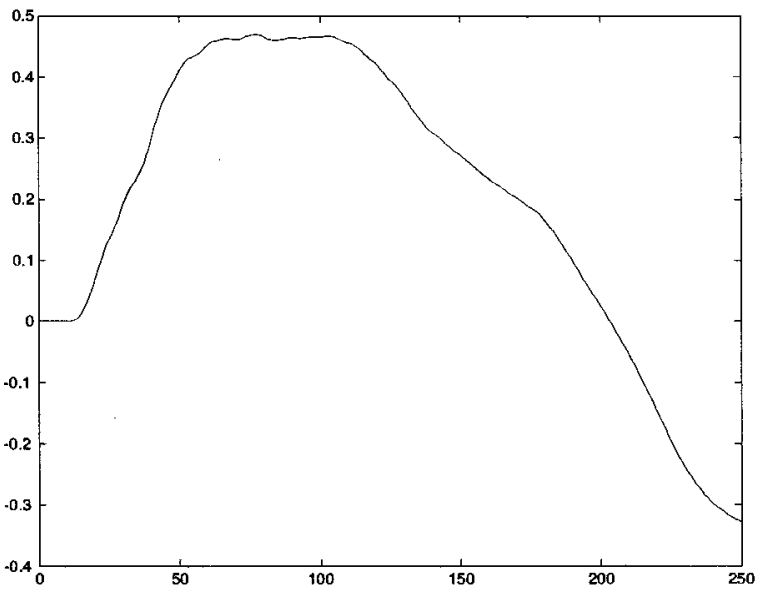

Figure 9. Errors in tracking the nominal state trajectory for the adaptive controller.

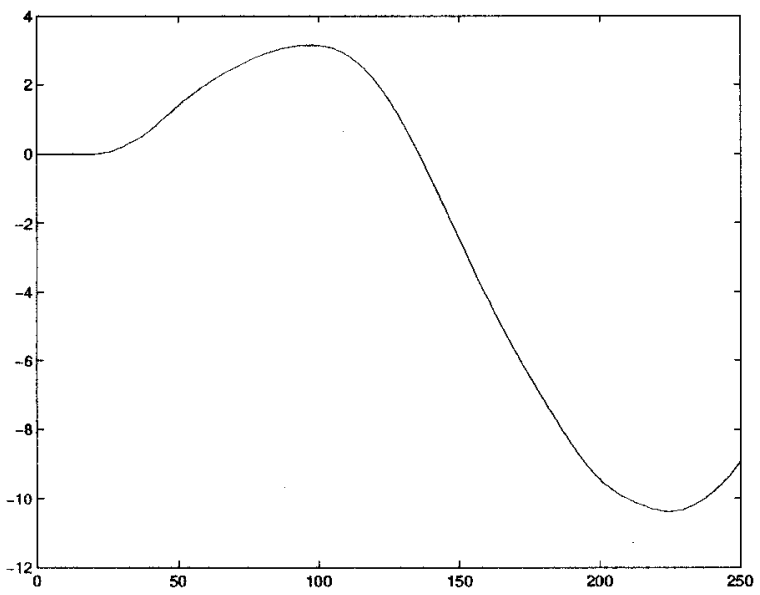

Figure 10. Erors in tracking the nominal state trajectory for the fixed controller.

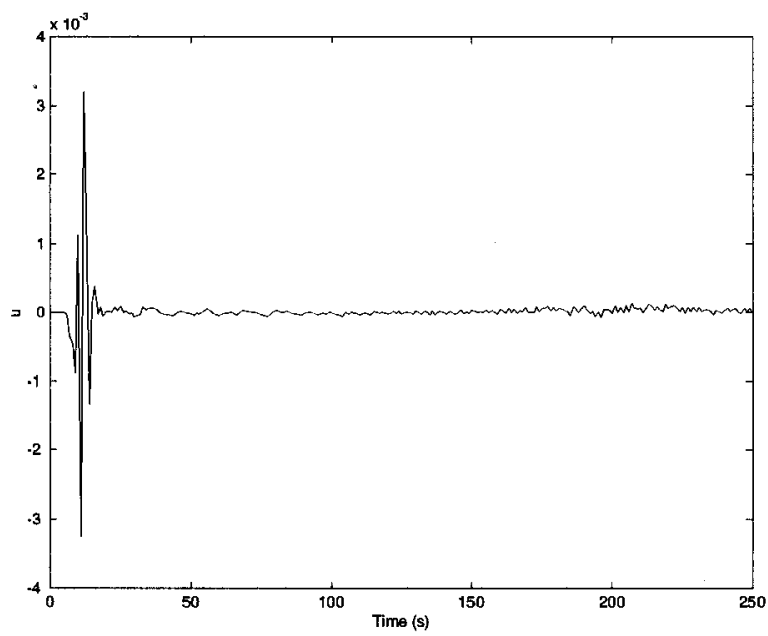

Figure 11. Correction of the input sequence for the adaptive controller.

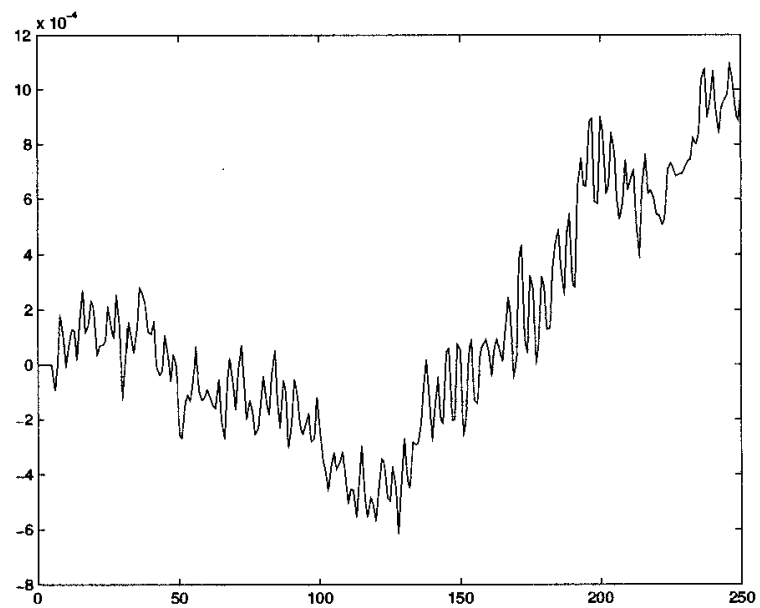

Figure 12. Correction of the input sequence for the fixed controller. 


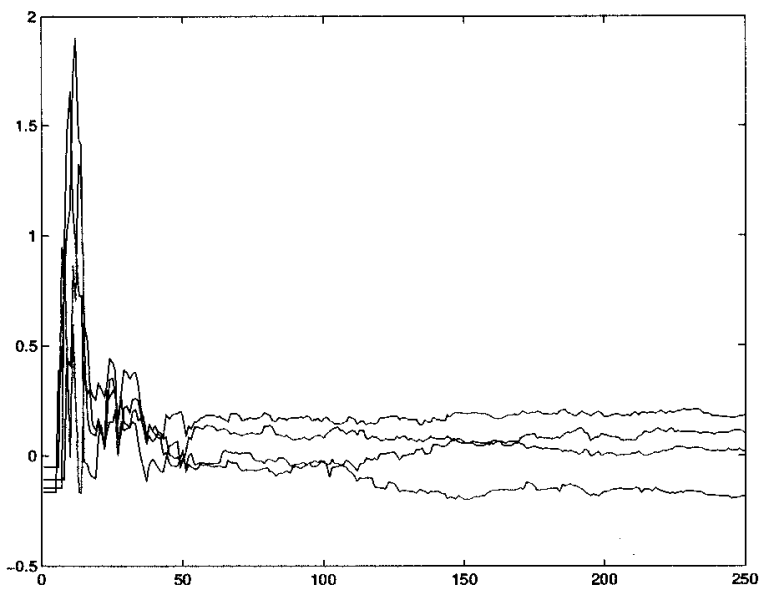

Figure 13. Estimated parameters of the polynomial $A$.

(28) is used to represent the nonlinear plant dynamics, the parameters of the chosen linear model have to be adjusted incessantly to approximate properly the behaviour of the nonlinear system concerned. Thus, the parameter convergence rate also represents the important issue for the overall adaptive controller performance. To judge this property, the estimated fourth-order ARMAX model is compared with the complete 12thorder linearized model obtained by direct linearization of the nonlinear plant model (28) around the corresponding operating point on the reference trajectory. Furthermore, since these non-stationary linear models are of different orders, their time constants are used as a performance measure. Figure 14 shows the time constant histories for the analysed models. Obviously, the adopted lower-order ARMAX model used for adaptive controller design approximates quite well with the time constant of the linearized non-stationary 12th-order

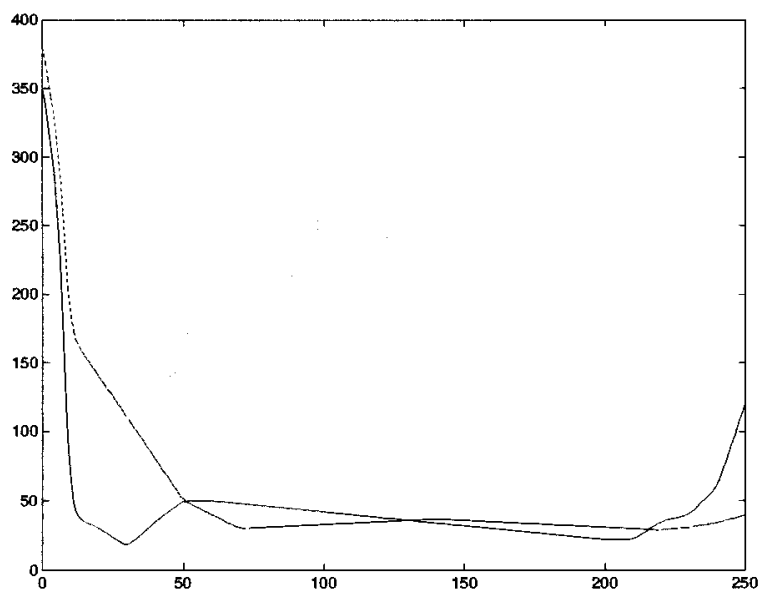

Figure 14. Time constant histories: linearized 12th-order model (solid) versus the fourth-order ARMAX model (dashed). model (which in turn approximates properly the nonlinear plant dynamics).

The most important process disturbance $d(k)$ during an aircraft flight is air turbulence. The turbulence models frequently used are the Dryden and von Karman model, and each consists of three velocity spectra $u, v$ and $w$, say $S_{u}(\Omega), S_{v}(\Omega)$ and $S_{w}(\Omega)$, corresponding to the three axis associated with a body-fixed coordinate system (Beal 1993). Here the frequency variable $\Omega(\mathrm{rad} / \mathrm{m})$ is spatial, so that the turbulence characteristics are independent of the aircraft speed $V$ in equation (26). Using the transformation s

$$
\omega(\mathrm{rad} / \mathrm{s})=\Omega(\mathrm{rad} / \mathrm{m}) V(\mathrm{~m} / \mathrm{s})
$$

and

$$
S_{u, v, w}(\omega)=S_{u, v, w}(\Omega) / V,
$$

one could generate the turbulence velocities directly in the time domain. Although the von Karman model gives a better approximation to the experimentally obtained air turbulence spectra, the Dryden model has a simpler analytical form, which is easier to implement (Beal 1993). Namely, the Dryden spectra is a rational function of frequency, so that the air turbulence velocities can be simulated directly by passing white noise through the corresponding linear filter, whose transfer function can be obtained by using the spectral factorization (Åström 1970, Åström and Wittenmark 1990, Kovačević and Djurovic 1999). Of course, the von Karman spectra, which represents the non-rational function of frequency, can be approximated by the rational one, and than the same procedure can be used for simulating these disturbance signals (Djurović et al. 2000). Finally, the turbulence effects can be incorporated by combining the generated random sequences with the appropriate elements of the dynamic system state vector in (29), i.e. $u_{\text {real }}=u+u_{\text {turbulence }} ; u_{\text {real }}=v+u_{\text {turbulence }}$ and $w_{\text {real }}=w+w_{\text {turbulence }}$. Figure 15 shows the simulated air-turbulence velocity $v$ in the time domain obtained by passing zero-mean white noise with unit variance through the transfer function

$$
G_{v}(s)=K\left(\prod_{i=1}^{3}\left(s-z_{i}\right) / \prod_{i=1}^{4}\left(s-p_{i}\right)\right),
$$

where

$$
\begin{aligned}
& K=0.04127, \quad z_{1}=-0.0157, \quad z_{2}=-0.0042, \\
& z_{3}=-0.00159, \quad p_{1}=-0.0210, \quad p_{2}=-0.005, \\
& p_{3}=-0.00182
\end{aligned}
$$

and $p_{4}=-0.001$ (Mišković 1999). Figures 16 and 17 shows the aerodynamic effects of the air-turbulence signals to the aircraft dynamics. 


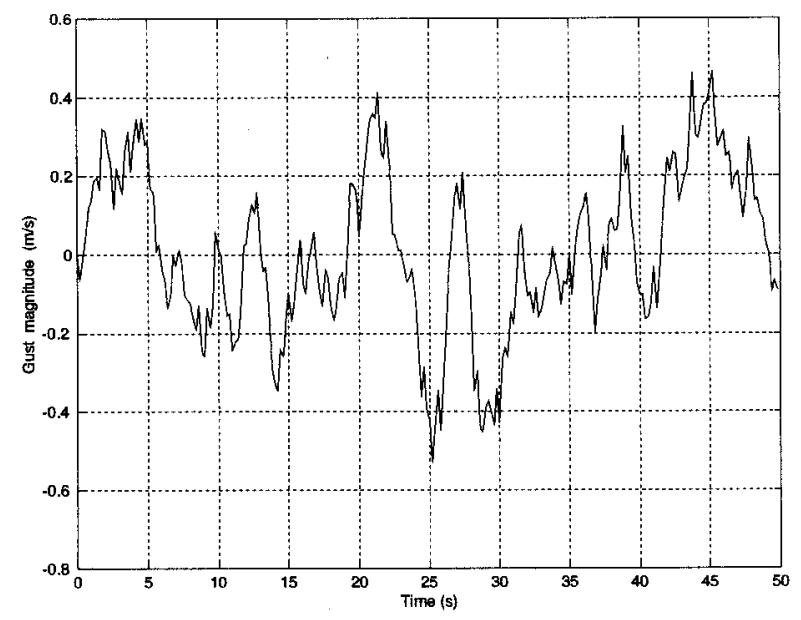

Figure 15. Air turbulence velocity versus sample.

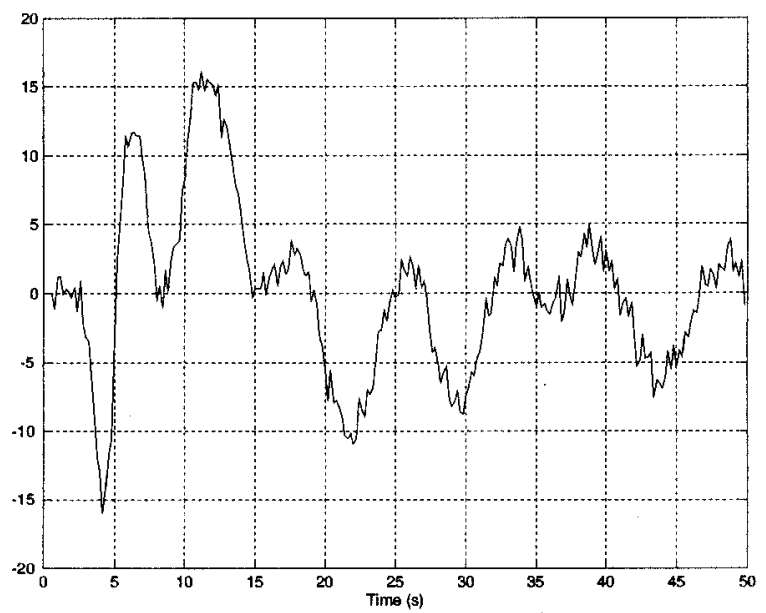

Figure 16. Errors in tracking the nominal state trajectory in the presence of air-turbulence signals.

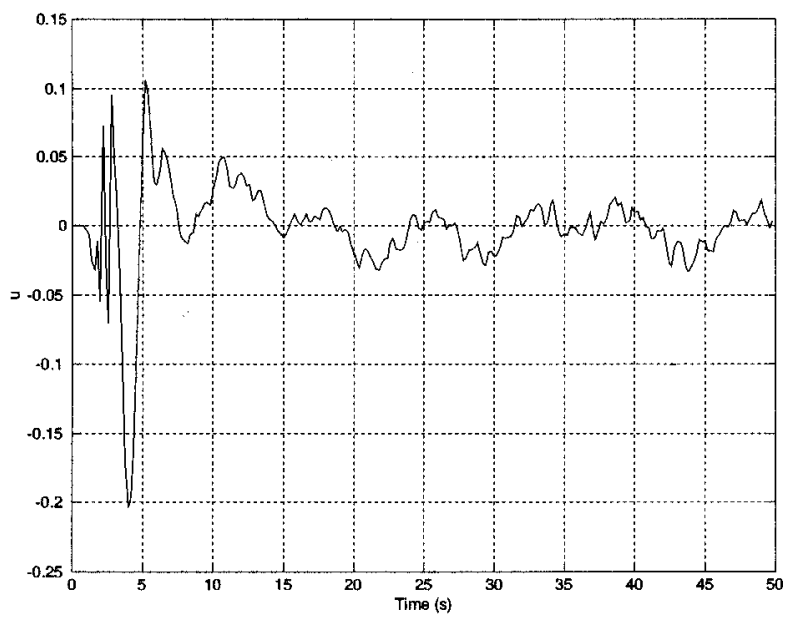

Figure 17. Correction of the input sequence in the presence of air-turbulence signals.
The obtained results have shown that the MSEV controller has good tracking performances and possesses rather good immunity towards stochastic disturbances. A possible way to cope with load disturbances has been considered by Mišković et al. (2000).

\section{Conclusion}

A form of self-tuning controller, named the modified minimum state error variance (MSEV) controller, has been proposed here. In contrast to the known MSEV approach from the literature, the proposed modifications enable tracking of a desired non-zero reference trajectory. A way of generating the reference trajectory by using numerical optimization has also been proposed. Moreover, the possibility of applying the modified MSEV approach for nonlinear and non-stationary plants control is analysed. The feasibility of the proposed approach for such applications is demonstrated through the example of aircraft control around the prespecified reference trajectory in the presence of process and observation disturbances. A comparison with the conventional fixed parameter controller was also included. A special emphasis is laid to the convergence of estimated time-varying model parameters as an important issue for the tracking capability of any adaptive controller. Time-constant history was used as a performance quality measure. The comparison of the calculated time-constant history for the high-order model obtained by linearizing the nonlinear plant model around the corresponding operating points on the reference trajectory and the same quantity calculated for the estimated lower order ARMAX model used for the adaptive controller design has shown that the parameter estimates are adapted properly to the nonlinear system dynamics.

The obtained results have shown that the proposed controller may represent an efficient tool for tracking a prespecified reference trajectory in the case of nonlinear and non-stationary system dynamics, as well as in the presence of stochastic disturbances with unknown statistic.

\section{Acknowledgment}

The authors are grateful to Professor P. J. Fleming and unknown reviewers for useful comments that helped to improve the original version of the manuscript.

\section{References}

Anderson, B. D. O., and Moore, J. B., 1971, Linear Optimal Control (Englewood Cliffs: Prentice Hall).

Anderson, B. D. O., and Moore, 1979, Optimal Filtering (Englewood Cliffs: Prentice Hall). 
Åström, K. J., 1970, Introduction to Stochastic Control Theory (New York: Academic Press).

Åström, K. J., and Wittenmark, B., 1989, Adaptive Control (New York: Addison-Wesley).

Åström, K. J., and Wittenmark, B., 1990, Computer Controlled Systems: Theory and Design (Englewood Cliffs: Prentice Hall).

BEAL, T. R., 1993, Digital simulation of atmospheric turbulence for Dryden and von Karman models. Journal of Guidance, 16, 132-138.

Clarke, D. W., and Gawthrop, P. J., 1975, A self-tuning controller. Proceedings of the IEE, Part D, 122, 929-934.

Clarke, D. W., and Gawthrop, P. J., 1979, Self-tuning control. Proceedings of the IEE, Part D, 126, 633-640.

Desai, P., and Mahanalabis, A. K., 1991, On the design of self-tuned regulators using state space approach. Control Theory and Advanced Technology., 7, 473-490.

Dixon, L. C. W., 1972, Nonlinear Optimization (London: English Universities Press).

Duurović, Ž. M., Mišković, L. M., and Kovačevíc, B. D., 2000, Simulation of air turbulence signal and its application. In 10th Mediterranean Electrotechnical Conference, vol. 2, Cyprus, pp. 847-850.
Goodwin, G. C., and Sin, K. S., 1984, Adaptive Filtering, Prediction and Control (Englewood Cliffs: Prentice Hall).

Grimble, M. J., 1981, A control-weighted minimum variance controller for non-minimum phase systems. International Journal of Control, 33, 751-762.

Koifman, M., and Merhav, S. J., 1991, Autonomously aided strapdown attitude reference systems. Journal of Guidance, 14, 433-448.

Kovačević, B. D., and Duurović, Ž. M., 1999, Fundamentals of Stochastic Signals, Systems and Estimation Theory with Worked Examples (Belgrade: Academic Mind).

Ljung, L., and SöDerström, T., 1983, Theory and Practice of Recursive Identification. (Cambridge, MA: MIT Press).

MišKović, L. M., 1999, Nonlinear and nonstationary system control using minimum state error variance strategy. Master's thesis, University of Belgrade.

Mišković, L. M., Duurović, Ž. M., and Kovačević, B. D., 2000, Nonlinear systems control using msev approach. Control and Intelligent Systems, 28, 110-117.

TsYPKIN, Ya Z., 1984, Foundations of the Informational Theory of Identification (Moscow: Nauka).

WARWICK, K., 1981, Self-tuning regulators: a state space approach. International Journal of Control, 33, 839-858. 